12

\title{
Образование и распад отрицательных ионов производных фталида
}

\author{
( Н.Л. Асфрандиаров, ${ }^{1}$ С.А. Пшеничнюк, ${ }^{1}$ Р.Г. Рахмеев, ${ }^{1,2}$ А.Н. Лачинов, ${ }^{1}$ В.А. Крайкин ${ }^{3}$ \\ ${ }^{1}$ Институт фризики молекул и кристаллов Уфимского научного центра РАН, \\ 450075 Уфра, Россия \\ ${ }^{2}$ Восточная экономико-юридическая академия, \\ 450071 Уфра, Россия \\ ${ }^{3}$ Уфимский институт химии РАН, \\ 450054 Уфра, Россия \\ e-mail: nail@anrb.ru
}

(Поступило в Редакцию 7 сентября 2017 г. В окончательной редакции 8 декабря 2017 г.)

\begin{abstract}
Методом масс-спектрометрии отрицательных ионов диссоциативного захвата электронов исследованы молекулы дифенилфталида и 3,3-дифенилфталид-4',4'-дикарбоновой кислоты. Измерены средние времена жизни молекулярных отрицательных ионов относительно выброса электрона. Величина сродства к электрону оценена в приближении Аррениуса и рассчитана методом DFT B3LYP/6-31G+(d).
\end{abstract}

DOI: $10.21883 /$ JTF.2018.07.46183.2487

\section{Введение}

Производные фталида являются мономерами для синтеза полиариленфталидов [1], тонкие пленки которых проявляют эффект зарядовой неустойчивости, что приводит к уменьшению их удельного сопротивления на несколько порядков величины [2]. Это весьма многообещающее явление с точки зрения создания молекулярных электрических переключателей и элементов энергонезависимой памяти. На сегодняшний день считается, что переход полимерной пленки в высокопроводящее состояние связан с тенденцией мономерного соединения к раскрытию пятичленного цикла [3], представленного на рис. 1. Здесь же приведена нумерация атомов в молекуле фталида.

Вполне очевидно, что подобный разрыв связи более вероятен в газовой фазе и в присутствии избыточного заряда, что в случае отдельной молекулы соответствует молекулярному аниону [4]. Позднее это было подтверждено в работе [5], в которой методами спектроскопии проходящих электронов (СПЭ) и масс-спектрометрии отрицательных ионов диссоциативного захвата электронов (МСОИ ДЗЭ) была исследована молекула фталида. Было установлено, что образование долгоживущих молекулярных отрицательных ионов (ОИ) происходит с разрывом связи $\mathrm{C}^{7}-\mathrm{O}^{8}$ через переходное состояние, лежащее в области энергии $0.65 \mathrm{eV}$ (рис. 2). Максимум интенсивности ионов $M^{-}$, где $M$ - молекула фталида, приходится на $0.75 \mathrm{eV}$. Время жизни ионов по отношению к автоотщеплению электрона при этой энергии и температуре камеры ионизации $80^{\circ} \mathrm{C}$ равно $\sim 100 \mu \mathrm{s}$.

Сродство к электрону $\left(E A_{a}\right)$ молекулы фталида с циклической структурой пятичленного гетероцикла равно по данным расчета DFT B3LYP/6-31G+(d) $0.17 \mathrm{eV}$ [5]. При раскрытии цикла энергия аниона фталида понижается на $0.32 \mathrm{eV}$. Поскольку раскрытие протекает путем преодоления потенциального барьера высотой $0.65 \mathrm{eV}$, „эффективное сродство“ открытой структуры оказывается равным $1.14 \mathrm{eV}$ [5]. В работе [5] было показано, что наблюдаемое время жизни ионов $M^{-}$фталида, равное $\sim 100 \mu \mathrm{s}$ (считается, что погрешность измерения этой величины лежит в пределах 10-15\%), хорошо воспроизводится в рамках простой аррениусовской модели $[6]$ :

$$
\tau_{a}=\tau_{0} \exp \left[\frac{N E A_{a}}{E A_{a}+N k T+\varepsilon}\right],
$$

где $\tau_{0}$ - характерное время движения иона по координате реакции; $N$ - число внутренних степеней свободы; $E A_{a}$ - адиабатическое сродство к электрону; $k$ - постоянная Больцмана; $T$ - эффективная температура молекулы-мишени; $\varepsilon$ - энергия захваченного электрона. Величина $\tau_{0}$ является параметром теории и варьирует от $10^{-13} \mathrm{~s}$ для производных нафтохинона [6] до $5 \cdot 10^{-13} \mathrm{~s}$ для производных нитробензола [7]. Расчет по формуле (1) при $\tau_{0}=5 \cdot 10^{-13} \mathrm{~s}$ и $T=80^{\circ} \mathrm{C}$ дает оценку $\tau_{a}=89 \mu \mathrm{s}$ (экспериментально измеренная величина составляет $100 \mu \mathrm{s}$ ) [5]. Практическое отсутствие сигнала $M^{-\cdot}$ при тепловых энергиях электронов обусловлено тем, что $\tau_{a}$ закрытой конформации молекулы фталида $\left(E A_{a}=0.17 \mathrm{eV}\right)$, согласно оценке $(1)$, не может превышать $10^{-9} \mathrm{~s}$. При этом анион не обладает достаточной колебательной энергией для разрыва связи $\mathrm{C}-\mathrm{O}$ и<smiles>[R]C([R])c1ccccc1C(=O)O</smiles>

Рис. 1. Схема раскрытия пятичленного цикла. 
перехода в более стабильную раскрытую конформацию, наблюдаемую при более высокой энергии электронов [5].

Целью настоящей работы является продолжение исследований производных фталида (Phthalide), a именно дифенилфталида (ДФФ, (DPP) $\mathrm{C}_{20} \mathrm{H}_{14} \mathrm{O}_{2}$ ) и 3,3-дифенилфталид-4', 4'-дикарбоновой кислоты (ДФФДКК, (DPPDCA) $\mathrm{C}_{22} \mathrm{H}_{14} \mathrm{O}_{6}$ ) методом МСОИ ДЗЭ, используемых в качестве мономеров для синтеза тонких пленок полимеров, обладающих необычными электрическими свойствами.

\section{Эксперимент}

Масс-спектры ОИ были получены на модифицированном масс-спектрометре МИ-1201 [8]. Суть эксперимента заключалась в следующем. Пучок электронов, коллимированный аксиальным магнитным полем, проходит через камеру ионизации, в которую испаряется исследуемое вещество. При этом в камере ионизации поддерживается давление $\sim 10^{-4} \mathrm{~Pa}$, обеспечивающее условия однократных столкновений [9]. Энергия электронов варьирует в характерном для резонансного захвата электронов диапазоне $0-15 \mathrm{eV}[9,10]$. Шкала энергии контролируется положением максимума пика ионов $\mathrm{SF}_{6}^{-} / \mathrm{SF}_{6}$, лежащим вблизи нуля [9]. Типичная ширина пика $\mathrm{SF}_{6}^{-}$на полувысоте составляет $\sim 0.4 \mathrm{eV}$ при токе электронов $1 \mu \mathrm{A}$, а точность измерения энергии максимума пиков ОИ равна $\pm 0.1 \mathrm{eV}$. Ионы под действием вытягивающего и выталкивающего потенциалов, проходя через систему щелей, попадают в область ускорения (ионный источник находится под потенциалом $-4 \mathrm{kV}$ ), а затем в первую бесполевую область. После этого они подвергаются селекции по массам в статическом магнитном массанализаторе. Разрешающая способность по массам составляет $\sim 1000$. Перед попаданием во вторичный электронный умножитель ионы проходят через вторую бесполевую область, в конце которой распложена система отклонения, необходимая для измерения средних времен жизни по отношению к автоотщеплению электрона.

Методика измерения среднего времени жизни ОИ относительно выброса электрона такова. Массспектрометр настраивают и фиксируют для регистрации молекулярных ОИ $M^{-\cdot}$ исследуемого вещества. На первом этапе эксперимента записывают кривую эффективного выхода $M^{-}$, т.е. ток молекулярных ОИ как функцию энергии электронов $\varepsilon$. Затем на систему отклонения перед входом в умножитель подают отклоняющий потенциал для удаления заряженной компоненты пучка исследуемых частиц и регистрируют сигнал нейтральных частиц $M^{0}$, образовавшихся при автоотщеплении электронов от $M^{-}$за время дрейфа молекулярных ОИ во второй бесполевой области. Для ионов $\mathrm{SF}_{6}^{-\cdot} / \mathrm{SF}_{6}$ $(m / e=146)$ время пролета бесполевой области до попадания в систему регистрации равно $6 \mu \mathrm{s}$. Эта величина известна с высокой точностью, поскольку определяется величиной ускоряющего потенциала на источнике ионов

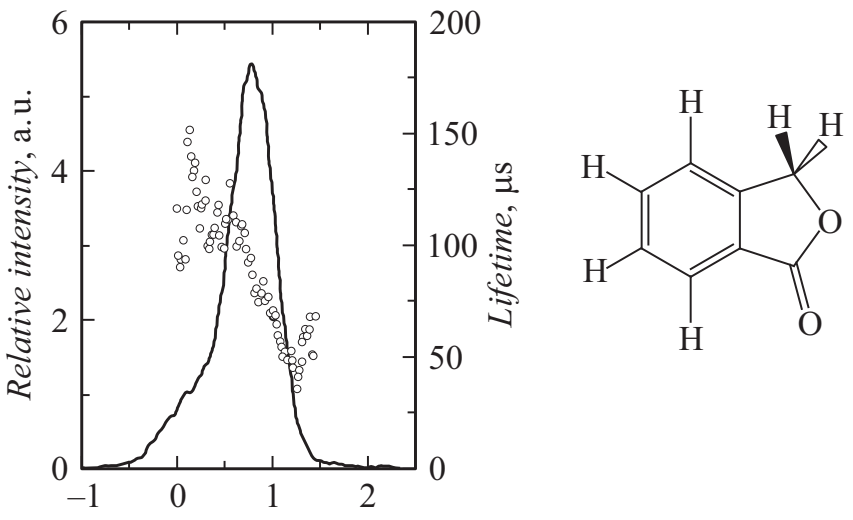

Рис. 2. Форма кривой эффективного выхода $M^{-}$из молекул фталида (сплошная линия) и зависимость его среднего времени жизни от энергии электронов (кружки) [5].

$(-4 \mathrm{kV})$ и расстоянием от выхода из магнита до входа в умножитель $(40 \mathrm{~cm})$. В предположении экспоненциального закона распада ОИ среднее время жизни ОИ равно [9]

$$
\tau_{a}=-\frac{t_{0}}{\ln \left(1-\frac{I_{n}}{I}\right)},
$$

где $t_{0}$ - время дрейфа ионов во второй бесполевой области, $I_{n}-$ сигнал нейтральных частиц $M^{0}$ (второй этап эксперимента), $I-$ суммарный сигнал $M^{0}$ и $M^{-\cdot}$ (первый этап измерений). Такой метод измерения $\tau_{a}$ был предложен Эдельсоном [11] и адаптирован Хвостенко для приборов со статическими массанализаторами [9]. Поскольку типичное время дрейфа ионов во второй бесполевой области составляет около $6 \mu \mathrm{s}$, верхним пределом измерений $\tau_{a}$ является величина около 5000-6000 $\mu \mathrm{s}$. В самом деле, если она превышает этот предел, отношение числа ионов, распавшихся за этот промежуток времени $I_{n}$ в формуле (2) к их исходному количеству $I$, будет порядка $0.1 \%$. Как следствие, ошибка измерений становится неприемлемой.

\section{Результаты и обсуждение}

Кривые эффективного выхода (КЭВ) ОИ при диссоциативном захвате электронов молекулами ДФФ и ДФФДКК приведены на рис. 3. В спектре ДФФ присутствуют лишь молекулярные ионы со средним временем автоотщепления $330 \mu \mathrm{s}$ при энергии $0.26 \mathrm{eV}$, соответствующей максимуму выхода ионов $M^{-}$(погрешность измерения около 10\%). Молекулы ДФФДКК образуют более долгоживущие молекулярные ОИ $(\sim 2800 \mu \mathrm{s})$, a максимум выхода $M^{-}$. лежит в области тепловых энергий электронов (рис. 3,b). Кроме того, ДФФДКК проявляет еще два канала распада $\left([M-\mathrm{H}]^{-}\right.$ и $\left.[M-\mathrm{COOH}]^{-}\right)$с крайне малой относительной интенсивностью (рис. $3, c, d)$. 


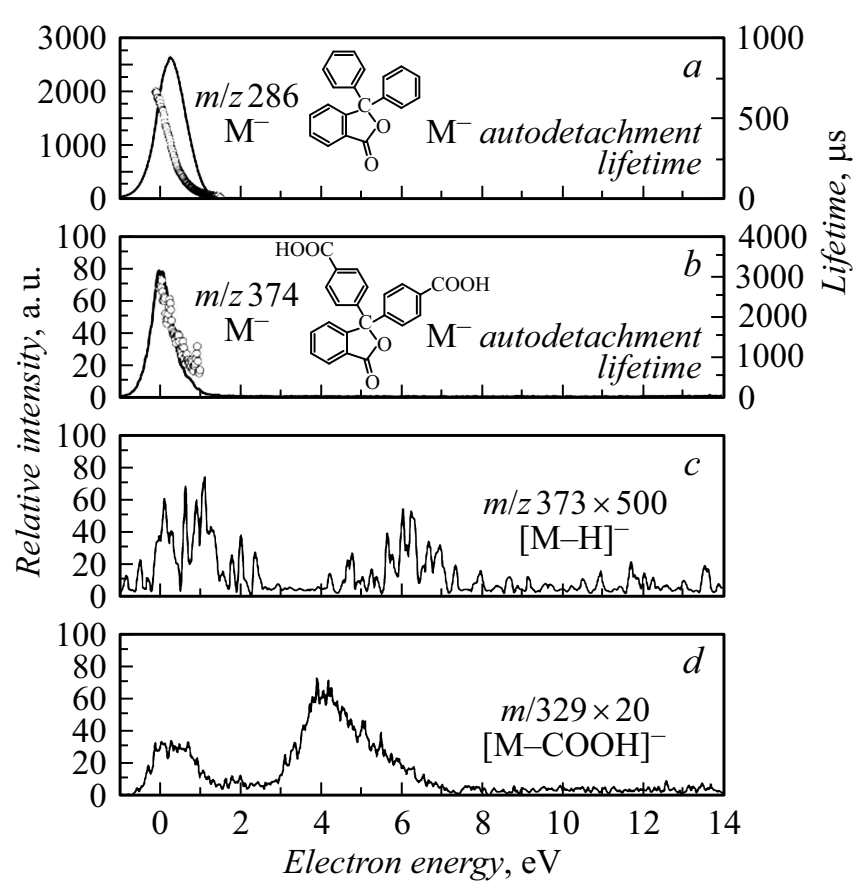

Рис. 3. Кривые эффективного выхода ОИ как функция энергии электронов для ДФФ $(a)$ и ДФФДКК $(b-d)$. Левая ордината относительная интенсивность ионов, правая ордината - среднее время жизни молекулярных ОИ.

В работе [5] было показано, что молекулярные ОИ фталида, образованные при энергии электронов $0.75 \mathrm{eV}$, находятся в раскрытой конформации пятичленного цикла и основном электронном состоянии. При этом эффективный потенциальный барьер, препятствующий возврату иона в циклическую конформацию, и, как следствие, выбросу электрона из молекулярного ОИ, составляет $\sim 1.14 \mathrm{eV}$. Проведем подобную оценку для исследуемых соединений. Оценка величины сродства к электрону в рамках приближения Аррениуса дает следующие результаты: $E A_{a}(Д Ф \Phi)=0.86 \mathrm{eV}$ (параметры: $N=102, \quad \varepsilon=0.26 \mathrm{eV}, \quad \tau_{0}=500 \mathrm{fs}, \quad T=$ $\left.=363 \mathrm{~K}, \tau_{a}=335 \mu \mathrm{s}\right) ; E A_{a}$ (ДФФДКК) $=0.92 \mathrm{eV}$ (параметры: $N=120, \varepsilon=0.26 \mathrm{eV}, \tau_{0}=500 \mathrm{fs}, \quad T=363 \mathrm{~K}$, $\left.\tau_{a}=2360 \mu \mathrm{s}\right)$. Во втором случае энергия электронов была выбрана аналогично молекуле ДФФ с целью унификации методики оценки.

Сравним эти величины с результатами расчетов DFT $\mathrm{B} 3 \mathrm{LYP} / 6-31 \mathrm{G}+(\mathrm{d})$. Для закрытой и открытой структур ДФФ $E A_{a}^{\text {close }}=0.41 \mathrm{eV}$ и $E A_{a}^{\text {open }}=0.96 \mathrm{eV}$; для ДФФДКК $E A_{a}^{\text {close }}=1.05 \mathrm{eV}$ и $E A_{a}^{\text {open }}=1.48 \mathrm{eV}$ соответственно. Характерно, что оценка сродства молекулы ДФФ по формуле Аррениуса (1) близка к предсказаниям квантовой химии для открытого цикла, а для ДФФДКК - для закрытой структуры. Это может быть обусловлено следующими причинами. Разрыв связи $\mathrm{C}-\mathrm{O}$ пятичленного цикла аниона фталида энергетически возможен из электронного состояния аниона с избыточным электроном на второй вакантной MO $\pi_{A}^{*}$-типа симметрии. По дан- ным спектроскопии проходящих электронов $[5,12,13]$ энергия вертикального захвата равна $0.57 \mathrm{eV}$, а резонанс в спектре ДЗЭ наблюдается при $0.75 \mathrm{eV}$. Расчеты энергий виртуальных MO (VOE - virtual orbital energy) методом DFT B3LYP/6-31G(d), масштабированные по формуле (3) из работы [14],

$$
\mathrm{SVOE}=0.8054 \cdot \mathrm{VOE}+1.211,
$$

либо по формуле (4), предложенной в работе [15]:

$$
\mathrm{SVOE}=0.8065 \cdot \mathrm{VOE}+0.9194,
$$

предсказывают следующий порядок следования и энергии (SVOE - scaled VOE) вакантных MO исследованных молекул (см. таблицу). Эмпирически полученные величины SVOE предсказывают энергии вертикального захвата электронов (VAE - vertical attachment energy), определяемые экспериментально методом СПЭ.

Сравнение расчетных энергий вакантных МО фталида [5] и ДФФ говорит о малом влиянии фенильных колец на положение первых трех орбиталей, однако их влияние на форму кривых эффективного выхода молекулярных ионов этих соединений весьма существенно. Действительно, во фталиде пик $M^{-\cdot}$ может быть представлен в виде суммы двух гауссовых пиков. Первый с максимумом, шириной и площадью $\varepsilon=0.19 \mathrm{eV}, w=0.62 \mathrm{eV}$, $A=0.78$; второй $-\varepsilon=0.8 \mathrm{eV}, w=0.45 \mathrm{eV}, A=2.97$ и соотношением площадей этих пиков $1: 3.8$. Очевидно, что оба пика образованы по механизму резонанса формы. В ДФФ аналогичная процедура разложения дает пики при $\varepsilon=0.23 \mathrm{eV}, w=0.65 \mathrm{eV}, A=2087$ и $\varepsilon=0.73 \mathrm{eV}$, $w=0.47 \mathrm{eV}, A=183$ с соотношением площадей $11.4: 1$. Наличие же на бензольных кольцах ДФФДКК заместителей СООН приводит к заметной стабилизации трех низших вакантных МО и смещению максимума $M^{-\cdot}$ в область тепловых энергий электронов. При этом параметры гауссовых пиков равны $\varepsilon=0.01 \mathrm{eV}, w=0.43 \mathrm{eV}$, $A=35$ и $\varepsilon=0.34 \mathrm{eV}, w=0.74 \mathrm{eV}, A=18.4$ с соотношением площадей $1.9: 1$, что позволяет интерпретировать первый из них как захват электрона на НВМО по механизму колебательно-возбужденного резонанса Фешбаха, а второй - на вторую вакантную МО $\pi_{A}^{*}$-типа по механизму резонанса формы. Сопоставление энергий и ширин пиков говорит о том, что первые пики во фталиде и дифенилфталиде сходны по параметрам со вторым пиком в ДФФДКК, что подтверждает интерпретацию их первоначального образования через резонанс формы. Первый пик молекулярных ионов в ДФКК при тепловой энергии электронов является „обычным“ колебательновозбужденным резонансом Фешбаха.

Вторым важным результатом расчетов анионных состояний ДФФ и ДФФДКК является то, что в отличие от аниона фталида, имеющего плоскую структуру с двумя углеродными атомами раскрытого пятичленного гетороцикла в $s p^{2}$-гибридизации, раскрытые конформации ДФФ и ДФФДКК являются неплоскими структурами с атомом углерода в седьмом положении в 
Энергии (eV) вакантных MO из расчетов DFT B3LYP/6-31G(d) VOE и их масштабированные (SVOE) по уравнению (3) величины, в скобках - по формуле (4)

\begin{tabular}{|c|c|c|c|c|}
\hline Соединение & Орбиталь & VOE & SVOE & VAE \\
\hline \multirow[t]{4}{*}{ Фталид } & $\pi_{\mathrm{O}}^{*} / \pi_{\mathrm{CO}}^{*}$ & 3.89 & $\begin{array}{c}4.34 \\
(4.06)\end{array}$ & 4.46 \\
\hline & $\pi_{\mathrm{CO}}^{*} / \pi_{\mathrm{S}}^{*}$ & 1.48 & $\begin{array}{c}2.40 \\
(2.11)\end{array}$ & 2.67 \\
\hline & $\pi_{\mathrm{A}}^{*}$ & -0.64 & $\begin{array}{c}0.70 \\
(0.40)\end{array}$ & 0.57 \\
\hline & $\pi_{\mathrm{S}}^{*} / \pi_{\mathrm{CO}}^{*}$ & -1.35 & $\begin{array}{c}0.13 \\
(-0.17)\end{array}$ & $0.2_{\mathrm{sh}}$ \\
\hline \multirow[t]{4}{*}{ Дифенилфталид } & $\pi_{\mathrm{O}}^{*} / \pi_{\mathrm{CO}}^{*}$ & 4.50 & $\begin{array}{c}4.84 \\
(4.55)\end{array}$ & \\
\hline & $\pi_{\mathrm{CO}}^{*} / \pi_{\mathrm{S}}^{*}$ & 1.551 & $\begin{array}{c}2.46 \\
(2.17)\end{array}$ & \\
\hline & $\pi_{\mathrm{A}}^{*}$ & -0.752 & $\begin{array}{c}0.61 \\
(0.31)\end{array}$ & \\
\hline & $\pi_{\mathrm{S}}^{*} / \pi_{\mathrm{CO}}^{*}$ & -1.354 & $\begin{array}{c}0.12 \\
(-0.17)\end{array}$ & \\
\hline $\begin{array}{c}\text { 3, 3-дифенилфталид- } \\
4^{\prime}, 4^{\prime} \text {-дикарбоновая } \\
\text { кислота }\end{array}$ & $\pi_{\mathrm{O}}^{*} / \pi_{\mathrm{CO}}^{*}$ & 3.16 & $\begin{array}{c}3.76 \\
(3.47)\end{array}$ & \\
\hline $\mathrm{HOOC}$ & $\pi_{\mathrm{CO}}^{*} / \pi_{\mathrm{S}}^{*}$ & 1.295 & $\begin{array}{c}2.25 \\
(1.96)\end{array}$ & \\
\hline & $\pi_{\mathrm{A}}^{*}$ & -0.851 & $\begin{array}{c}0.53 \\
(0.23)\end{array}$ & \\
\hline 'Ó & $\pi_{\mathrm{S}}^{*} / \pi_{\mathrm{CO}}^{*}$ & -1.726 & $\begin{array}{c}-0.18 \\
(-0.47)\end{array}$ & \\
\hline
\end{tabular}



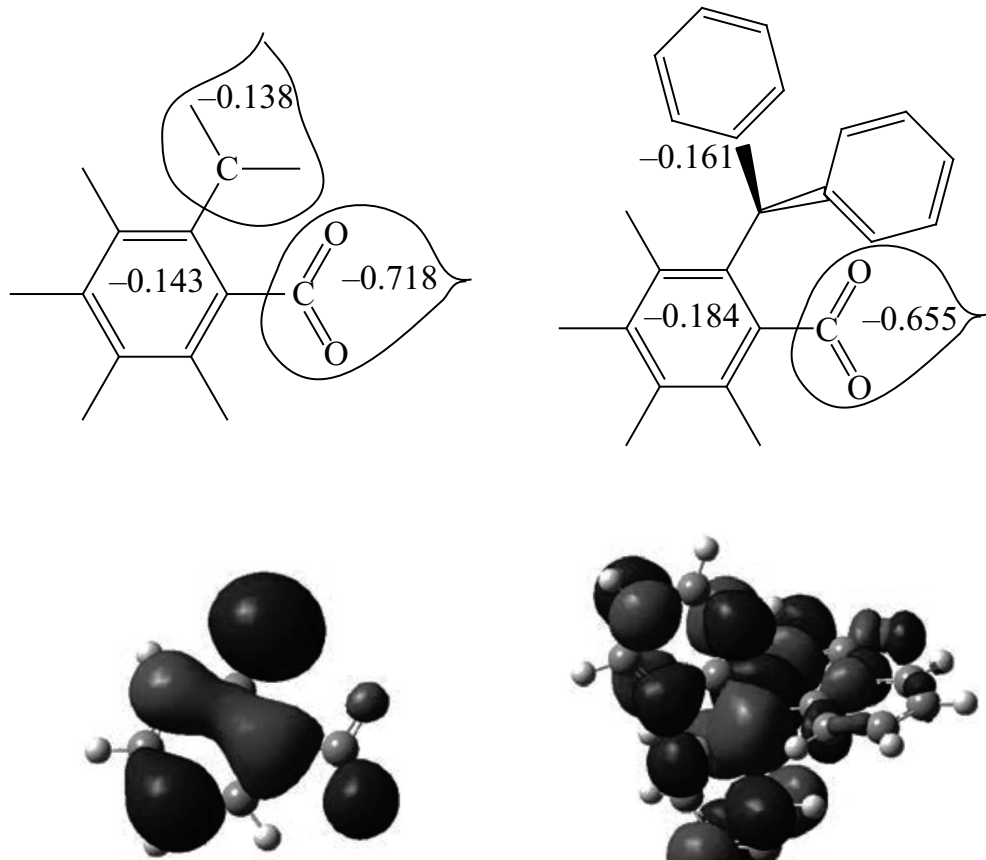

Phthalide $s p^{2}$

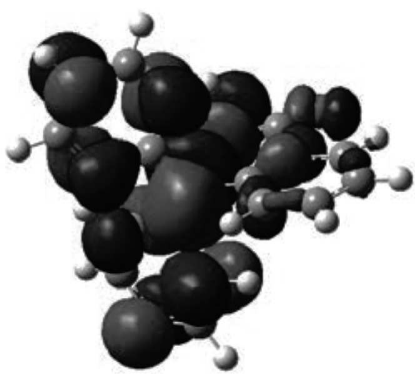

DPP $s p^{3}$
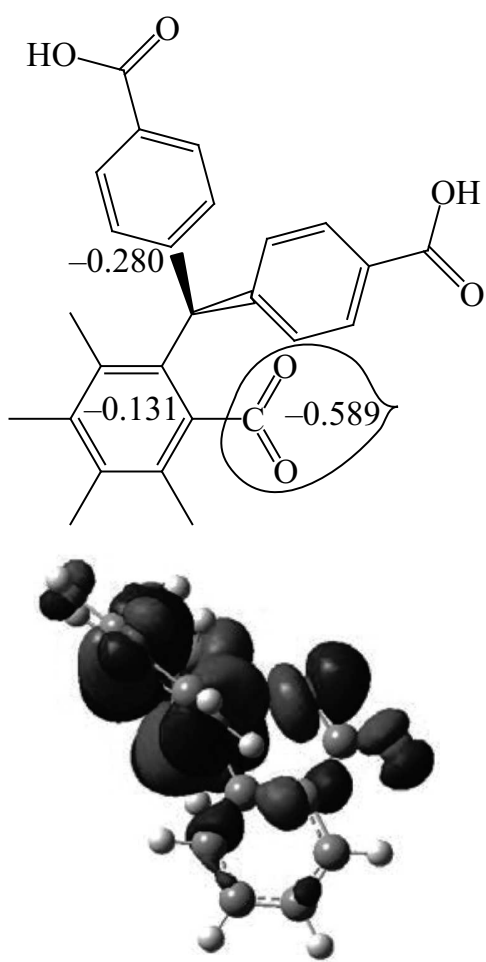

DPPDCA $s p^{3}$

Рис. 4. Распределение избыточного заряда (сверху), вид однократно занятой МО анионов производных фталида и гибридизация углеродного атома при раскрытии цикла (внизу).

$s p^{3}$-гибридизации (рис. 4). Причина вполне очевидна фенильные заместители геометрически не могут расположиться в плоскости фталидного остова. На рисунке также представлено распределение избыточного заряда анионов в ряду фталид-ДФФ-ДФФДКК. В этом ряду наблюдается отчетливая тенденция перераспределения избыточного заряда с карбоксильной группы на фенильные кольца заместителей.

Результаты DFT-расчетов говорят о том, что делокализация избыточного электрона по фенильным кольцам заместителей в анионах ДФФ и ДФФДКК выгодней, нежели нахождение его на НВМО $\pi_{\mathrm{S}}^{*} / \pi_{\mathrm{CO}}^{*}$-типа (расчет оптимальной геометрии аниона в циклической конформации) (см. рисунки в таблице). При этом первоначально захват электрона происходит на НВМО молекулы, т. е. в соответствии с теоремой Купманса $[16,17]$.

\section{Выводы}

Измерены и интерпретированы спектры ДЗЭ двух производных фталида. Оценено и проанализировано влияние заместителей на энергии вакантных МО и локализацию избыточного заряда в анионах. Механизмы захвата электронов в области низких энергий электронов интерпретированы с помощью DFT-расчетов. Для выяснения деталей механизмов захвата электрона и особенностей стабилизации молекулярных ОИ относи- тельно выброса электрона производными фталида, в частности энергетики раскрытия пятичленного цикла, необходимы дальнейшие исследования молекул этого ряда.

Работа частично поддержана грантами РФФИ № 15-02-02809, 15-29-05786 и 18-03-00179.

\section{Список литературы}

[1] Салазкин С.Н., Шапошникова В.В., Мачуленко Л.Н., Гилева Н.Г., Крайкин В.А., Лачинов А.Н. // Высокомолекулярные соединения. Сер. А. 2008. Т. 50. № 3. С. 399-418.

[2] Лачинов А.Н., Жеребов А.Ю., Корнилов В.М. // Письма в ЖЭТФ. 1990. Т. 52. Вып. 2. С. 742-745.

[3] Лачинов А.Н., Воробьева Н.В. // УФН. 2006. Т. 176. № 12. C. 249-266.

[4] Зыков Б.Г., Васильев Ю.В., Фалько В.С., Лачинов А.Н., Хвостенко В.И., Гилева Н.Г. // Письма в ЖЭТФ. 1996. Т. 64. Вып. 6. С. 402-406.

[5] Asfandiarov N.L., Pshenichnyuk S.A., Vorob'ev A.S., Nafikova E.P., Lachinov A.N., Kraikin V.A., Modelli A. // J. Chem. Phys. 2015. Vol. 142. P. 174308. http://dx.doi.org/10.1063/1.4919631

[6] Asfandiarov N.L., Pshenichnyuk S.A., Vorob'ev A.S., Nafikova E.P., Elkin Y.N., Pelageev D.N., Koltsova E.A., Modelli A. // Rapid Commun. Mass Spectrom. 2014. Vol. 28. P. 1580-1590. http://dx.doi.org/10.1002/rcm.6934 
[7] Asfandiarov N.L., Pshenichnyuk S.A., Vorob'ev A.S., Nafikova E.P., Modelli A. // Rapid Commun. Mass Spectrom. 2015. Vol. 29. P. 910-912. http://dx.doi.org/10.1002/rcm.7162

[8] Pshenichnyuk S.A., Vorob'ev A.S., Modelli A. // J. Chem. Phys. 2011. Vol. 135. P. 184301. http://dx.doi.org/10.1063/1.3658372

[9] Хвостенко В.И. Масс-спектрометрия отрицательных ионов в органической химии. М.: Наука, 1981. 159 с.

[10] Илленбергер Е., Смирнов Б.М. // УФН. 1998. Т. 168. № 7. C. 731 .

[11] Edelson D., Griffiths J.E., McAfee K.B. // J. Chem. Phys. 1962. Vol. 37. P. 917-918. http://dx.doi.org/10.1063/1.1733191

[12] Jordan K., Burrow P.D. // Chem. Rev. 1987. Vol. 87. P. $557-588$.

[13] Воробьев А.С., Пшеничнюк С.А., Асббандиаров Н.Л., Набикова Е.П. // ЖТФ. 2014. Т. 84. Вып. 9. С. 17-25. http://journals.ioffe.ru/jtf/2014/09/page-17.html.ru

[14] Modelli A. // Phys. Chem. Chem. Phys. 2003. Vol. 5. P. $2923-$ 2930. http://dx.doi.org/10.1039/B304083F

[15] Scheer A.M., Burrow P.D. // J. Phys. Chem. B. 2006. Vol. 110. P. 17751. http://dx.doi.org/10.1021/jp0628784

[16] Koopmans T. // Physica. 1934. Vol. 1-6. P. 104. https://doi.org/10.1016/S0031-8914(34)90011-2

[17] Simons J., Jordan K.D. // Chem. Rev. 1987. Vol. 87. P. 535-555. http://dx.doi.org/10.1021/cr00079a004 